\title{
Metformin extended-release versus metformin immediate-release for adults with type 2 diabetes mellitus: a systematic review and meta-analysis of randomized controlled trials
}

\section{Aedrian A. Abrilla ( $\sim$ aaabrilla@up.edu.ph )}

College of Medicine, University of the Philippines Manila https://orcid.org/0000-0003-1938-3403

\section{A. Nico Nahar I. Pajes}

Office of the Chief Resident, Department of Medicine, Philippine General Hospital, University of the Philippines Manila https://orcid.org/0000-0003-2368-9923

\section{Cecilia A. Jimeno}

Office of the Chair, Department of Pharmacology and Toxicology, College of Medicine, University of the Philippines Manila https://orcid.org/0000-0002-7658-0123

\section{Systematic Review}

Keywords: extended-release, glycemic control, serum lipid control, metformin, systematic review, type 2 diabetes mellitus

Posted Date: December 2nd, 2021

DOI: https://doi.org/10.21203/rs.3.rs-137066/v3

License: (c) (i) This work is licensed under a Creative Commons Attribution 4.0 International License. Read Full License

Version of Record: A version of this preprint was published at Diabetes Research and Clinical Practice on April 19th, 2021. See the published version at https://doi.org/10.1016/j.diabres.2021.108824. 


\section{Abstract}

This systematic review aimed to compare the efficacy and tolerability of metformin extended-release (MXR) and the conventional metformin immediate-release (MIR) in adults with type 2 diabetes mellitus (T2DM). PubMed, the Cochrane Library and ClinicalTrials.gov, from database inception to 15 October 2020, and other sources were searched for randomized controlled trials (RCTs) that compared equal daily doses of MXR and MIR in adults with T2DM. Random-effects model meta-analysis was performed to obtain pooled mean difference (MD) of change from baseline for continuous outcomes (glycemic and serum lipid control and anthropometrics) and risk ratio (RR) for dichotomous outcomes (gastrointestinal and serious adverse events). Statistical analysis involving 9 published RCTs with 2609 subjects revealed that MIR was associated with better HbA1c lowering (MD 0.09\% [95\% confidence interval, $0.02 \%, 0.17 \%$ ]) and serum lipid control except LDL-C lowering, while MXR reduced only the cumulative incidence of dyspepsia (RR $0.58[0.34,0.98])$. MXR and MIR were similar in all other considered outcomes. The use of MXR over MIR among adults with T2DM was associated with statistically worse but likely clinically insignificant $\mathrm{HbA} 1 \mathrm{c}$ lowering, similar plasma glucose lowering, and minimal improvement of metformin intolerance. This information may guide patient-physician discussions in choosing between the two formulations.

\section{Introduction}

Diabetes mellitus (DM) is a chronic progressive metabolic disease and a global threat to health. It currently afflicts more than 463 million people worldwide, at least $90 \%$ of which are type 2 DM (T2DM) cases [1]. DM is the $15^{\text {th }}$ leading cause of total years of life lost in 2017 [2] and its economic impact is approximately $2 \%$ of the global gross domestic product [3]. As the burden of this disease continues to worsen, developing and implementing integrative strategies for its prevention and control is thus a matter of urgency in all levels of care.

Comprehensive medical care for diabetes mellitus includes optimized and individualized glycemic goal and therapeutic plan [4]. As lifestyle modification (nutrition, physical activity, psychosocial care, and selfmanagement, education and support [4]) often alone fails to establish and sustain optimal glycemic control [5], pharmacotherapy forms an important component of management [6]. In this regard, with more than sixty years of clinical experience and evidentiary support of efficacy, safety and tolerability in numerous T2DM trials [7], the biguanide metformin has been recommended for use as a first-line therapy, together with lifestyle modification, in managing the disease [8].

Unfortunately, a significant number of patients prescribed with metformin cannot tolerate the conventional immediate-release formulation at recommended doses because of gastrointestinal (GI) adverse events (AEs), leading to reduced treatment adherence $[9,10]$. Furthermore, this drug preparation notably demonstrates a lack of dose proportionality with increasing doses, resulting to decreased bioavailability [11]. As all these may contribute to untoward outcomes ranging from poor glycemic control to hospitalizations [12], intolerance to metformin immediate-release (MIR) is a pressing clinical 
concern. One supposed strategy to address MIR intolerance is alternatively prescribing using metformin extended-release (MXR). MXR slowly delivers the active component of the drug in the upper GI tract and allows for once-daily dosing and is thus designed to reduce GI AEs and to improve adherence [6,7,9-12]. Furthermore, its bioavailability is not adversely affected either by variations in daily dosing frequency or meal intake [7,12]. Since its regulatory approval in Canada and in the United States 15 years ago [6], MXR has been marketed as an equally efficacious yet more GI-tolerable alternative to MIR.

Nevertheless, a systematic review has yet to be undertaken to validate assumptions regarding MXR prescription to adults with T2DM in avoiding or reducing metformin intolerance. The purpose of this study was thus to compare MXR and MIR in terms of efficacy and tolerability in the randomized controlled trial (RCT) setting involving adults with T2DM.

\section{Methods}

\subsection{Protocol, Registration and Reporting}

The methods of this systematic review and meta-analysis were pre-specified in a protocol that was registered in the International Prospective Register of Systematic Reviews (PROSPERO) with registration number CRD42019148008 (Supplement 1). Reporting was done in accordance to Preferred Reporting Items for Systematic Review and Meta-Analysis (PRISMA) guidelines. Activities and decisions at any point were independently conducted and made by two reviewers (A.A.A. and A.N.N.I.P.), with disagreements resolved through discussion or the senior author (C.A.J.). The deviations to the original protocol and justifications are indicated in Supplement 2.

\subsection{Eligibility Criteria}

RCTs that compared equal daily dosing of MXR and MIR on separate trial arms for at least 12 weeks which involved adults diagnosed with T2DM were considered. Studies with other interventions together with MXR and MIR were considered if the concomitants were provided equally in terms of dose, exposure or intensity in both arms. No restriction was set regarding publication status, year, and language. Studies that explicitly involved subjects with end-stage organ diseases were excluded.

\subsection{Outcomes}

The primary outcomes were glycemic control parameters, such as glycated hemoglobin or hemoglobin A1c (HbA1c), fasting plasma glucose (FPG) and post-prandial plasma glucose after 2 hours (PPG), and GI AEs such as abdominal distension, abdominal discomfort or pain, constipation, diarrhea, dyspepsia, flatulence, and nausea and vomiting. Secondary outcomes were serum lipid control parameters, such as serum triglycerides (Tg), total cholesterol (TC), high-density lipoprotein-cholesterol (HDL-C) and lowdensity lipoprotein-cholesterol (LDL-C), anthropometric parameters such as weight and body mass index (BMI), and serious adverse events, such as hypoglycemia and lactic acidosis.

\subsection{Search Strategy and Study Selection}


PubMed, the Cochrane Library and ClinicalTrials.gov were searched for records of studies. A sensitive search strategy was implemented, including "metformin," "extended release," "gastric retention," "slow release," and "sustained release" (Supplement 3). Google searching was also performed for records not indexed in the specified databases. There was neither date, language nor study design restriction in the search. Reference lists of selected studies were also screened from eligible reports and relevant reviews. Unpublished results, print-only journals and conference proceedings were also sought by contacting the authors.

Items in the initial search were scanned through titles and abstracts to remove irrelevant papers and duplicates. The criteria for selection and exclusion of the remaining articles were implemented and documented. Figure 1 illustrates the outcome of the selection process through the PRISMA flow diagram.

\subsection{Data Collection and Analysis}

Data were independently extracted from included articles using a pre-piloted data collection form. Quality assessment was performed in each selected study through the Cochrane Collaboration risk of bias (RoB) tool [13]. A study was then deemed to have an overall low risk (low risk in all RoB domains), overall unclear risk (unclear risk in at least one domain and high risk in no domain) or overall high risk (high risk in at least one domain). Quantitative synthesis through meta-analysis with a Mantel-Haenszel random effects model was also performed with results reported, with 95\% confidence interval $(\mathrm{Cl})$ and p-value (statistical significance set at $p<0.05)$, as risk ratio (RR) for dichotomous outcomes and mean difference (MD) of change from baseline for continuous outcomes. Heterogeneity was declared if detected in at least one of the two tests, $\chi^{2}$ ( $p \leq 0.1$ indicating significant heterogeneity) and $\mathrm{I}^{2}$ (at least $50 \%$ indicating substantial heterogeneity). Subgroup analyses were performed to explore possible sources of heterogeneity from differences in daily total metformin dose. Publication bias was ascertained through funnel plot using the outcome change from baseline $\mathrm{HbA} 1 \mathrm{c}$. Sensitivity analyses were conducted based on risk of bias (high risk, unclear risk, low risk) per particular domain and eliminating one included study at a time. Review Manager version 5.4 (Cochrane Collaboration) was utilized.

\subsection{Imputations}

In the absence of response from authors of included studies for queries on data, the following strategies were performed to address insufficient or missing continuous outcome values. The MD and standard deviation (SD) of change from baseline values were re-calculated using reported confidence intervals, means, and SDs or standard errors of baseline and endpoint measures. SDs of calculated change from baseline values were computed using the following formula (Equation 1):

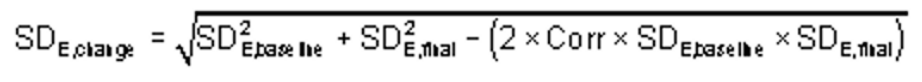

where Corr was set conservatively at 0.5 . When the SD of interest was not reported or was reported but deemed unreasonably wide (with the particular study subsequently contributing too little weight in the 
analyses relative to its contribution to the pooled sample size) by author consensus, a validated summary statistic-level prognostic imputation method was performed utilizing undisputed SDs from included studies (Equation 2) [14]:

$$
\mathrm{SD}=\frac{\sum_{i=1}^{k} S D i}{k}
$$

where the numerator refers to the summation of undisputed SDs and the denominator refers to the number of undisputed SDs.

\section{Results}

\subsection{Search Results}

A total of 1272 database records (415 from PubMed, 738 from the Cochrane Library and 119 from ClinicalTrials.gov) were obtained through the latest systematic search performed on 15 October 2020, and another one was found through the Google search engine. After removing 471 records as duplicates, the rest were subjected through title and abstract screening wherein 790 were excluded. Nine full-text articles of RCTs, that directly compared MXR and MIR, satisfied the eligibility criteria (Table 1) and were included in both the qualitative and quantitative syntheses. On the other hand, 1 unpublished clinical trial $[15]$ and 2 full-text articles $[16,17]$ were excluded because of unavailable study results, metformin daily doses were adjusted based on gastrointestinal tolerance on a per-patient basis (and thus were virtually not equal between the two arms), and concomitant non-metformin anti-T2DM medications were not controlled, respectively (Figure 1).

\subsection{Study Characteristics}

Six trials were explicitly double-blinded involving patients and caregivers [18-23] while 2 were open-label [24,25]; it was not apparent in one study whether blinding at any level was performed [26]. Six studies had formal run-in period [18,19,22-25], 3 of which having a passive washout period (2 [22], 4 [23] and 6 [18] weeks, respectively). In the 3 studies with active run-in periods, 2 provided MIR in both treatment arms (for a total of either 2 [24] or 10 [19] weeks), while 1 provided MXR and MIR in the respective treatment arms for 2 weeks [25]. Two studies had explicit post-treatment period, one lasted for an additional 12 weeks wherein the MXR or MIR daily dose was increased by 500 mg if $\mathrm{HbA} 1 \mathrm{c} \geq 8 \%$ [19], while the other comprised of an additional 2 weeks without treatment on both arms [25].

All except three trials $[21,22,26]$ were multicentric, with the number of sites ranging from 3 to 148 $[11,19,20,23-25]$. All were published at or after the year 2003 ( 4 before and 5 after 2010), written in English and published in peer-reviewed journals. Six trials were at least partially sponsored by pharmaceutical companies [18-20,23-25]. Three trials have registered their protocol in ClinicalTrials.gov $[20,23,25]$, while only two indicated the registration number in the final publication and appended the study results in the registration entry $[23,25]$. 
A total of 2609 participants (mean [SD]: 54.3 [11.5] years; $45.9 \%$ female), with a median (range) of 207 (40-750), in the outpatient setting from 14 countries were randomized (Table 2) and followed up for a median (range) of 16 (12-24) weeks. Majority of the participants were Caucasian, while four studies $[20,22,24,25]$ explicitly enrolled patients of East Asian origin. No included trial specified the utilized T2DM diagnostic criteria in the report. Only three reports presented a justification of the trial sample size and statistical power $[20,23,25]$. Only one trial reported the prevalence of a comorbidity (hypertension) among its participants [24].

There was substantial variation in the total daily MXR and MIR dose given in the studies such as 500 $\mathrm{mg} /$ day (combined with the sulfonylurea glimepiride in equivalent dosing in both arms) in 1 study [20], $1000 \mathrm{mg} /$ day in 2 studies [19,21], $1500 \mathrm{mg} /$ day in 2 studies [18,24] and $2000 \mathrm{mg} /$ day in 4 studies $[22,23,25,26]$. Two studies had an additional arm (MXR daily dose $500 \mathrm{mg}$ greater than the two equivalent arms [19] and MXR daily dose 1000 mg less than the two equivalent arms [26]). Notably, 1 study had 2 MXR arms with total metformin daily dose equal to each other (MXR was provided once daily in one arm and twice daily in the other) and to the MIR arm [18]; the 2 MXR arms were thus pooled for quantitative analyses. Six studies had dose build-up weeks within the treatment period, which lasted from one week to one month $[18,21-23,25,26]$. The formal duration of treatment also varied across studies: 12 weeks in 4 trials [19,22,24,26], 16 weeks in 2 trials [20,25], 18 weeks in 1 trial [21] and 24 weeks in 2 trials [18,23]. The frequency of follow-up for assessment of outcomes ranged from 1 to 4 weeks in seven trials $[18,19,21,22,24-26]$, while two trials performed the same only at baseline and at endpoint (16 weeks [20] and 24 weeks [23], respectively).

\subsection{Imputations}

Four studies $[18,19,24,26]$ only reported separate baseline and endpoint means \pm SDs; thus, Equation 1 was applied to generate MD $\pm S D$ change from baseline values. Equation 2 was used to generate SDs for one study [18] that reported values that were unanimously considered by the authors as too wide (resulting to a relative weight in the analyses that was too small compared to the contributed sample size, see Supplement 4) and for another [21] where the SDs for mean change from baseline HbA1c could not be obtained.

\subsection{Risk of Bias and Publication Bias}

All included trials were judged to have unclear risk of selection bias (random sequence generation and allocation concealment) as none of them presented adequate relevant details for assessment. Two studies $[23,26]$ were considered to have an overall unclear risk of bias. An overall high risk of bias was noted for the remaining 7 studies [18-22,24,25], 2 of which were explicitly unblinded [24,25] (Figure 2 and Supplement 5).

The generated funnel plot (Figure S6) using the primary outcome of change from baseline HbA1c showed funnel plot asymmetry. However, since this plot involves less than 10 studies, other sources of asymmetry 
such as selective outcome reporting, poor methodological quality, true heterogeneity, artefactual elements and chance cannot be reliably ruled out [27].

\subsection{Primary Outcomes}

In terms of glycemic control parameters, MIR was associated with better mean $\mathrm{HbA} 1 \mathrm{c}$ lowering from baseline $(0.09 \%[95 \% \mathrm{Cl}, 0.02 \%, 0.17 \%])$, with neither significant nor substantial heterogeneity $\left(\chi^{2} p=0.39\right.$, $\mathrm{I}^{2}=6 \%$ ), than MXR (Table 3 and Figure S7.1). Conditions eliminating Schwartz et al. (2006) [18], with imputed SDs being used, in the sensitivity analyses abolished this finding (Table S8.1). The difference in mean FPG lowering from baseline (Table S7.2) was not statistically significant $(0.03 \mathrm{mmol} / \mathrm{L}[95 \% \mathrm{Cl}$, $-0.14 \mathrm{mmol} / \mathrm{L}, 0.19 \mathrm{mmol} / \mathrm{L}]$ ) between the two arms, also without significant nor substantial heterogeneity $\left(X^{2} p=0.37, I^{2}=8 \%\right)$. No statistically significant finding was noted for both outcomes in subgroup analyses by daily metformin dose. While the difference in mean PPG lowering from baseline (Table S7.3) was also not statistically significant $(0.60 \mathrm{mmol} / \mathrm{L}[95 \% \mathrm{Cl},-0.22 \mathrm{mmol} / \mathrm{L}, 1.43 \mathrm{mmol} / \mathrm{L})$, only 3 studies $[20,24,25]$ reported this outcome and the heterogeneity was notably significant and substantial $\left(X^{2} p=0.08, I^{2}=61 \%\right)$. Statistically better PPG lowering was noted for MIR at the total daily metformin dose of $1500 \mathrm{mg}$ [24]. On sensitivity analyses, both FPG and PPG findings were robust (Tables S8.2 and S8.3).

In terms of GI AEs, MXR reduced dyspepsia (RR 0.58 [95\% Cl, 0.34, 0.98], 4 studies [18,19,25,26]), with neither significant nor substantial heterogeneity $\left(X^{2} p=0.95, I^{2}=0 \%\right)$, compared to MIR (Figure S7.4). Sensitivity analyses revealed that conditions removing at least 1 of 3 studies $[18,19,26]$ eliminated this finding (Table S8.4). MXR was not associated with reduced risk of abdominal discomfort or pain (RR 1.29 [95\% Cl, 0.82, 2.02], 6 studies [18,19,21,23-25]), abdominal distension (RR 1.05 [95\% Cl, 0.54, 2.03], 1 study [25]), constipation (RR 0.99 [95\% Cl, 0.29, 3.37], 1 study [24]), diarrhea ( $R R 0.84$ [95\% Cl, 0.57, 1.26], 7 studies [18,19,21,23-26]), flatulence (RR 0.61 [95\% Cl, 0.23, 1.58], 4 studies [19,24-26]), and nausea and vomiting (RR 0.90 [95\% Cl, 0.63, 1.30], 6 studies [18,19,21,23-25]). Heterogeneity was neither significant nor substantial $\left(X^{2} p>0.1, l^{2}<50 \%\right)$ in all dichotomous outcomes reported by more than one study (Figures S7.5 to S7.8). Subgroup analyses for all GI AEs did not reveal statistically significant findings in all daily metformin doses. These findings were also found to be robust on sensitivity analyses (Tables S8.5 to S8.8).

\subsection{Secondary Outcomes}

In terms of change from baseline in serum lipid control parameters, MIR was associated with improved mean Tg lowering $(0.18 \mathrm{mmol} / \mathrm{L}[95 \% \mathrm{Cl}, 0.07 \mathrm{mmol} / \mathrm{L}, 0.29 \mathrm{mmol} / \mathrm{L}], 6$ studies [18,19,21-24]), better mean TC lowering $(0.10 \mathrm{mmol} / \mathrm{L}[95 \% \mathrm{Cl}, 0.00 \mathrm{mmol} / \mathrm{L}, 0.21 \mathrm{mmol} / \mathrm{L}], 6$ studies $[18,19,21-24])$ and increased mean HDL-C elevation $(0.04 \mathrm{mmol} / \mathrm{L}$ [95\% Cl, $0.01 \mathrm{mmol} / \mathrm{L}, 0.07 \mathrm{mmol} / \mathrm{L}], 5$ studies $[18,19,21,23,24])$, with neither significant nor substantial heterogeneity $\left(\chi^{2} p \geq 0.41,1^{2}=0 \%\right)$ in all cases, compared to MXR (Tables S7.11 to S7.13). In subgroup analyses, statistically significant change was found only for HDL-C elevation at metformin 2000 mg/day in favor of MIR. Sensitivity analyses show 
that the statistical significance of these findings were not robust when (1) studies with unclear or high risk for performance and/or reporting bias were removed, (2) at least one of the studies, except the smallest [21], were eliminated and (3) elimination of studies with unclear or high risk for performance, detection, attrition or reporting bias was performed, respectively (Tables S8.9 to S8.11). Mean lowering of LDL-C (-0.03 mmol/L [95\% Cl, $-0.12 \mathrm{mmol} / \mathrm{L}, 0.06 \mathrm{mmol} / \mathrm{L}]$, 5 studies $[18,19,21,23,24])$ was not significantly different between the two arms (Figure S7.14), with neither significant nor substantial heterogeneity $\left(X^{2} p=0.41, I^{2}=0 \%\right)$. The finding was robust on sensitivity analysis (Table S8.12).

Mean change in baseline weight $(0.09 \mathrm{~kg}[95 \% \mathrm{Cl},-0.47 \mathrm{~kg}, 0.64 \mathrm{~kg}], 3$ studies [21-23]) and BMI $(-0.10$ $\mathrm{kg} / \mathrm{m}^{2}\left[95 \% \mathrm{Cl},-0.82 \mathrm{~kg} / \mathrm{m}^{2}, 0.61 \mathrm{~kg} / \mathrm{m}^{2}\right], 2$ studies [22,24]) were also not statistically different in the two arms (Figures S7.15 and S7.16), with neither significant nor substantial heterogeneity $\left(\chi^{2} p \geq 0.75,1^{2}=\right.$ $0 \%$ ). Two studies $[20,25]$ reported hypoglycemic episodes (Figure S7.17) that were statistically similar between both arms (RR $1.38[95 \% \mathrm{Cl}, 0.79,2.42])$, with neither significant nor substantial heterogeneity $\left(\chi^{2}\right.$ $p=0.26, I^{2}=22 \%$ ). Robust results were elicited after sensitivity analyses of these outcomes (Tables $\mathrm{S} 8.13$ to $\mathrm{S} 8.15)$. None of the included studies reported incidence of lactic acidosis.

\section{Discussion}

\subsection{Summary, Interpretation and Context of Results}

MXR was associated with statistically inferior $\mathrm{HbA1c}$ lowering and with reduced cumulative incidence of dyspepsia compared to MIR. Both interventions were found to be equivalent in terms of shorter-term glycemic control (FPG and PPG lowering), cumulative incidence of all other GI AEs, anthropometrics, and LDL-C lowering. Interestingly, MIR led to better Tg and TC lowering as well as HDL-C elevation than MXR.

MXR first received regulatory approval for use as a therapeutic agent for adult-onset T2DM in 2005, with accumulated evidence purporting similar glycemic control and improved GI tolerability compared to the conventional MIR formulation [6]. While subsequent RCTs comparing MXR and MIR in equal daily doses individually reported general glycemic control equivalence [20-26], a portion of these studies that reported cumulative incidence of AEs demonstrated similarity in GI tolerability profile as well [21,23-25]. Moreover, the measures of dispersion reported in Schwartz et al. (2006) [18] - whose results were integral to the regulatory approval of MXR a year prior to its publication - were uncharacteristically wide in comparison to those reported in other included studies. When used directly in the meta-analysis, these measures decreased considerably the weight of the trial relative to its contribution in the pooled sample size. This compelled us to use imputation Equation 2 [14] for all continuous outcomes in this trial where it provided information ( $\mathrm{HbA} 1 \mathrm{c}, \mathrm{FPG}, \mathrm{Tg}, \mathrm{TC}, \mathrm{LDL}-\mathrm{C}$ and $\mathrm{HDL}-\mathrm{C}$ ). The status of statistical significance in the findings between the non-imputed and imputed scenarios was the same for FPG, Tg, LDL-C and HDL-C, while the difference in $\mathrm{HbA} 1 \mathrm{c}$ and TC lowering became statistically significant in favor of MIR when the imputation was performed (Supplement 4). 
The results suggest that most included trials were likely to be individually underpowered in ascertaining the difference in glycemic control between MXR and MIR. On the other hand, it remains to be seen whether the statistically better HbA1c lowering noted for MIR in this study translates to tangible difference in morbidity or mortality outcomes compared to MXR, as there exists no study that compares "hard" outcomes between the two. Nevertheless, the current body of evidence suggests that this particular finding is unlikely to be clinically significant $[28,29]$. While comparing cumulative incidences of AEs with MXR and MIR suggests that the former only minimally improved metformin intolerance relative to the latter, it is possible that comparing cumulative incidences are not sensitive enough to gauge the difference in metformin tolerability between the two formulations. Incidence rates and incidence rate ratios, rather than cumulative incidences and RRs, may be able to tease out clinically relevant findings in GI AE burden between these two formulations, if the difference in tolerability truly exists. As the frequency and severity of GI AEs has been suggested to subside as a function of time that metformin is taken [10], future RCTs comparing MXR and MIR should aim to comprehensively describe and report AE occurrence associated with these interventions beyond cumulative incidence. In terms of significant secondary outcomes, the finding that MIR lowered Tg by $5.99 \mathrm{mg} / \mathrm{dL}$ to $26.05 \mathrm{mg} / \mathrm{dL}$ and TC by $0.06 \mathrm{mg} / \mathrm{dL}$ to 7.92 $\mathrm{mg} / \mathrm{dL}$ compared to MXR can be considered clinically relevant for at least some cases (especially those with $\mathrm{Tg}$ and/or TC level at or near the risk factor thresholds for these parameters). The clinical significance of improved HDL-C increase with MIR compared to MXR, however, remains uncertain, as it is yet to be convincingly shown that such elevation by pharmacologic agents, among patients without genetic perturbations (e.g., deficiency or excess in serum levels) in the metabolism of the said lipoprotein, improves clinical outcomes [30].

\subsection{Limitations}

While the included trials were conducted in 14 different countries, majority of the participants were Caucasian in origin. The pooled age estimate of the participants (mean [SD] of 54.3 [11.5] years) suggests that the findings of this meta-analysis would likely not be applicable in relatively younger and older patients with T2DM, both growing subsets of the population afflicted with the disease. Majority of the included studies are considered to have an overall high risk of bias and the reporting quality of one efficacy (PPG) and several tolerability parameters of primary interest is arguably superficial (cumulative incidence rather than incidence rate without a measure of severity in all studies) and incomplete (only 3 studies reporting PPG, and relatively few or no GI AEs presented in many included studies). The pooled estimates also reflect findings across 4 daily metformin doses. The impact of comorbidities and concomitant intake of non-T2DM medications in the findings could also not be ascertained as these data were not specified in most included trials. Findings from one reportedly completed study that would have been eligible for inclusion in the systematic review were not included due to unavailability [15], hence not all of the existing evidence was analyzed. Imputation strategies were also implemented which may not adequately estimate the actual aggregate measures observed from the involved trials. Finally, individual patient data meta-analysis was not and could not be performed which would otherwise allow consistent definition of exposures and outcomes as well as proper adjustments for confounders [31]. 


\subsection{Conclusions}

In this meta-analysis of RCTs comparing MXR and MIR, MXR was statistically associated with worse but likely clinically insignificant $\mathrm{HbA} 1 \mathrm{c}$ lowering and with reduced cumulative incidence of dyspepsia. On the other hand, intake of MIR led to improved Tg and TC lowering as well as HDL-C elevation compared to MXR. In terms of FPG and PPG reduction, cumulative incidence of $\mathrm{Gl}$ and serious AEs other than dyspepsia, LDL-C lowering, and anthropometrics, the difference between the two formulations is not statistically significant. While the findings of this study remain to be validated and the definitive clinical implications yet to be elucidated by future trials, these data may be utilized by physicians in discussing metformin-related treatment options with their adult patients with T2DM especially in the setting of significant GI intolerance. Additional head-to-head high-quality RCTs, with protocols informed by the study-level flaws and quality assessments mentioned in this study, that will also provide individual patient-level data are needed. In particular, the use of incidence rates rather than cumulative incidence especially for safety and tolerability related outcomes should be considered. Investigations that aim to mechanistically prove or disprove the findings of this study are of interest, including the noted difference of both formulations in serum lipid control parameters. Difference in cost-effectiveness and adherence rates between these two formulations may also be explored.

\section{Declarations}

\section{FUNDING}

Funding was neither sought nor received in making this study.

\section{AUTHOR CONTRIBUTIONS}

All authors contributed to the conception and design of the systematic review. A.A.A. wrote the first draft of the protocol and the full manuscript. A.A.A. and A.N.N.I.P. independently performed the systematic review processes under supervision from C.A.J. who also rendered final decision in instances of disagreement between the two. All authors (1) critically revised the protocol and manuscript and (2) approved the final version for publication.

\section{DECLARATION OF COMPETING INTEREST}

The authors declare no conflict of interest.

\section{References}

[1] Saeedi P, Petersohn I, Salpea P, Malanda B, Karuranga S, Unwin N, et al. Global and regional diabetes prevalence estimates for 2019 and projections for 2030 and 2045: results from the International Diabetes Federation Diabetes Atlas, 9th edition. Diabetes Res Clin Pract 2019;157:107843. https://doi.org/10.1016/j.diabres.2019.107843. 
[2] Roth GA, Abate D, Abate KH, Abay SM, Abbafati C, Abbasi N, et al. Global, regional, and national agesex-specific mortality for 282 causes of death in 195 countries and territories, 1980-2017: a systematic analysis for the Global Burden of Disease Study 2017. Lancet 2018;392:1736-88. https://doi.org/10.1016/S0140-6736(18)32203-7.

[3] Bommer C, Sagalova V, Heesemann E, Manne-Goehler J, Atun R, Bärnighausen T, et al. Global economic burden of diabetes in adults: projections from 2015 to 2030. Diabetes Care 2018;41:963-70. https://doi.org/10.2337/dc17-1962.

[4] Powers AC, Niswender KD, Rickels MR. Diabetes mellitus: management and therapies. In: Jameson Jl, Fauci AS, Kasper DL, Hauser SL, Longo DL, Loscalzo J, editors. Harrison's Principles of Internal Medicine. 20th ed., New York, USA: McGraw-Hill Education; 2018, p. 2859.

[5] Nathan DM, Buse JB, Davidson MB, Heine RJ, Holman RR, Sherwin R, et al. Management of hyperglycemia in type 2 diabetes: a consensus algorithm for the initiation and adjustment of therapy. Diabetes Care 2006;29:10. https://doi.org/10.2337/dc06-9912.

[6] Schwartz SL, Wu JF, Berner B. Metformin extended release for the treatment of type 2 diabetes mellitus. Expert Opin Pharmacother 2006;7:803-9. https://doi.org/10.1517/14656566.7.6.803.

[7] Fujita Y, Inagaki N. Metformin: new preparations and nonglycemic benefits. Curr Diab Rep 2017;17:5. https://doi.org/10.1007/s11892-017-0829-8.

[8] Davies MJ, D’Alessio DA, Fradkin J, Kernan WN, Mathieu C, Mingrone G, et al. Management of hyperglycemia in type 2 diabetes, 2018. A consensus report by the American Diabetes Association (ADA) and the European Association for the Study of Diabetes (EASD). Diabetes Care 2018;41:2669-701. https://doi.org/10.2337/dci18-0033.

[9] Jabbour S, Ziring B. Advantages of extended-release metformin in patients with type 2 diabetes mellitus. Postgrad Med 2011;123:15-23. https://doi.org/10.3810/pgm.2011.01.2241.

[10] Bonnet F, Scheen A. Understanding and overcoming metformin gastrointestinal intolerance. Diabetes Obes Metab 2017;19:473-81. https://doi.org/10.1111/dom.12854.

[11] Schwartz SL, Gordi T, Hou E, Cramer M, Heritier M, Cowles VE. Clinical development of metformin extended-release tablets for type 2 diabetes: an overview. Expert Opin Drug Metab Toxicol 2008;4:123543. https://doi.org/10.1517/17425255.4.9.1235.

[12] Chacra AR. Evolving metformin treatment strategies in type-2 diabetes: from immediate-release metformin monotherapy to extended-release combination therapy. Am J Ther 2014;21:198-210. https://doi.org/10.1097/MJT.0b013e318235f1bb.

[13] Higgins JPT, Altman DG, Gotzsche PC, Juni P, Moher D, Oxman AD, et al. The Cochrane Collaboration's tool for assessing risk of bias in randomised trials. BMJ 2011;343:d5928. 
https://doi.org/10.1136/bmj.d5928.

[14] Ma J, Liu W, Hunter A, Zhang W. Performing meta-analysis with incomplete statistical information in clinical trials. BMC Med Res Methodol 2008;8:56. https://doi.org/10.1186/1471-2288-8-56.

[15] Metformin SR tablet (DuLeNing) and glucophage in patients with type 2 diabetes (CQM-2016). ClinicalTrialsGov 2019. https://clinicaltrials.gov/ct2/show/NCT03039075.

[16] Derosa G, D’Angelo A, Romano D, Maffioli P. Effects of metformin extended release compared to immediate release formula on glycemic control and glycemic variability in patients with type 2 diabetes. Drug Design Dev Ther 2017;11:1481-8. https://doi.org/10.2147/DDDT.S131670.

[17] Kim J-D, Park C-Y, Cha B-Y, Ahn KJ, Kim IJ, Park KS, et al. Comparison of adherence to glimepiride/metformin sustained release once-daily versus glimepiride/metformin immediate release BID fixed-combination therapy using the medication event monitoring system in patients with type 2 diabetes. Clin Ther 2018;40:752-61. https://doi.org/10.1016/j.clinthera.2018.04.002.

[18] Schwartz S, Fonseca V, Berner B, Cramer M, Chiang Y-K, Lewin A. Efficacy, tolerability, and safety of a novel once-daily extended-release metformin in patients with type 2 diabetes. Diabetes Care 2006;29:759-64. https://doi.org/10.2337/diacare.29.04.06.dc05-1967.

[19] Fujioka K, Pans M, Joyal S. Glycemic control in patients with type 2 diabetes mellitus switched from twice-daily immediate-release metformin to a once-daily extended-release formulation. Clin Ther 2003;25:515-29. https://doi.org/10.1016/S0149-2918(03)80093-0.

[20] Hwang Y-C, Kang M, Ahn CW, Park JS, Baik SH, Chung DJ, et al. Efficacy and safety of glimepiride/metformin sustained release once daily vs. glimepiride/metformin twice daily in patients with type 2 diabetes. Int J Clin Pract 2013;67:236-43. https://doi.org/10.1111/ijcp.12071.

[21] Ghorpade V, Hiray R, Daswani B, Ghongane B. Comparison of conventional and sustained-release formulation of metformin in type 2 diabetics. Int J Basic Clin Pharmacol 2016;5:274-80. https://doi.org/10.18203/2319-2003.ijbcp20160654.

[22] Hsieh C-H, He C-T, Lee C-H, Wu L-Y, Hung Y-J. Both slow-release and regular-form metformin improve glycemic control without altering plasma visfatin level in patients with type 2 diabetes mellitus. Metabolism 2007;56:1087-92. https://doi.org/10.1016/j.metabol.2007.03.018.

[23] Aggarwal N, Singla A, Mathieu C, Montanya E, Pfeiffer AFH, Johnsson E, et al. Metformin extendedrelease versus immediate-release: an international, randomized, double-blind, head-to-head trial in pharmacotherapy-naïve patients with type 2 diabetes. Diabetes Obes Metab 2018;20:463-7. https://doi.org/10.1111/dom.13104.

[24] Gao H, Xiao W, Wang C, Zhang J, Yang Y, Yang J, et al. The metabolic effects of once daily extendedrelease metformin in patients with type 2 diabetes: a multicentre study: Effects of metformin XR in 
patients with type 2 diabetes. Int J Clin Pract 2008;62:695-700. https://doi.org/10.1111/j.1742-

1241.2008.01733.x.

[25] Ji L, Liu J, Yang J, Li Y, Liang L, Zhu D, et al. Comparative effectiveness of metformin monotherapy in extended release and immediate release formulations for the treatment of type 2 diabetes in treatmentnaïve Chinese patients: Analysis of results from the CONSENT trial. Diabetes Obes Metab 2018;20:100613. https://doi.org/10.1111/dom.13190.

[26] Hameed M, Khan K, Salman S, Mehmood N. Dose comparison and side effect profile of metformin extended release versus metformin immediate release. J Ayub Med Coll Abbottabad 2017;29:225-9.

[27] Sterne JAC, Egger M, Moher D. Addressing reporting biases. In: Higgins JPT, Green S, editors. Cochrane Handbook for Systematic Reviews of Interventions, England, UK: John Wiley \& Sons Ltd.; 2008, p. 310-4.

[28] Little RR, Rohlfing CL. The long and winding road to optimal HbA1c measurement. Clin Chim Acta 2013;418:63-71. https://doi.org/10.1016/j.cca.2012.12.026.

[29] Svensson E, Baggesen LM, Johnsen SP, Pedersen L, Nørrelund H, Buhl ES, et al. Early glycemic control and magnitude of $\mathrm{HbA} 1 \mathrm{c}$ reduction predict cardiovascular events and mortality: population-based cohort study of 24,752 metformin initiators. Diabetes Care 2017;40:800-7. https://doi.org/10.2337/dc162271.

[30] März W, Kleber ME, Scharnagl H, Speer T, Zewinger S, Ritsch A, et al. HDL cholesterol: reappraisal of its clinical relevance. Clin Res Cardiol 2017;106:663-75. https://doi.org/10.1007/s00392-017-1106-1.

[31] Thomas D, Radji S, Benedetti A. Systematic review of methods for individual patient data metaanalysis with binary outcomes. BMC Med Res Methodol 2014;14:79. https://doi.org/10.1186/1471-228814-79.

\section{Tables}

Due to technical limitations, the tables are only available as a download in the supplemental files section.

\section{Figures}

\section{Figure 1}

PRISMA Flow Diagram of the literature search. 
Figure 2

Risk of bias assessment graph (A) and summary (B).

\section{Supplementary Files}

This is a list of supplementary files associated with this preprint. Click to download.

- Tables.docx

- Supplementarycontent20201020.docx

- PRISMA2009checklist20201020.doc 